\title{
Differences in the expression of telomerase and prostate-specific membrane antigen in non-advanced prostatic cancer
}

\author{
Anna Gasinska ${ }^{1}$, Elzbieta Luczynska ${ }^{2}$, Waclaw Wilk ${ }^{3}$, Anna Cichocka ${ }^{1}$ \\ ${ }^{1}$ Department of Applied Radiobiology, Centre of Oncology, Maria Sklodowska-Curie Memorial \\ Institute, Cracow, Poland \\ ${ }^{2}$ Department of Radiology, Centre of Oncology, Maria Sklodowska-Curie Memorial Institute, \\ Cracow, Poland \\ ${ }^{3}$ Department of Pathology, Centre of Oncology, Maria Sklodowska-Curie Memorial Institute, \\ Cracow, Poland
}

\begin{abstract}
Early recognition of prostate cancer (PC) based on biological markers could be helpful in identification of differences in benign and malignant lesions and facilitate further precise indication for more aggressive treatment. Therefore, the aim of our study was to assess expression of hTERT (human telomerase reverse transcriptase, a catalytic subunit of telomerase) and prostate-specific membrane antigen (PSMA), both considered to be markers of tumor aggressiveness. 140 low advanced PC specimens from patients who underwent radical prostatectomy were studied. Protein expression was assessed immunohistochemically on tumor sections and expressed as labeling index (LI), i.e. the percentage of positively stained cells. In case of telomerase, only nuclear staining and in case of PSMA, membrane and cytoplasmic staining, were considered as positive. The mean age of the patients was $62.9 \pm 6.2$ years. There were $75(53.6 \%)$ well differentiated tumors (Gleason score

6), $52(37.1 \%)$ moderately differentiated tumors (Gleason score of 7$)$ and $13(9.3 \%)$ poorly differentiated tumors (Gleason score 8-10). The mean pre-operative serum PSA was $9.9 \pm \mathrm{SE} 5.5 \mathrm{ng} / \mathrm{mL}$, and the mean LI were $18.0 \pm 1.5 \%$ and $44.1 \pm 1.9 \%$, for hTERT and PSMA, respectively. With increase of pathological tumor stage and tumor grade statistically significant increase of PSA serum concentration $(\mathrm{P}<0.011)$ and PSMA $(\mathrm{P}<0.004)$ expression was noticed, however, for expression of telomerase the relation was opposite one. The observed in higher pTNM stages and tumor grades decrease in nuclear expression of hTERT was caused by translocation of the subunit to the cytoplasm, what may indicate extranuclear telomerase activity independent of telomere lengthening, hence, it cannot be considered as a marker of malignancy. Higher PSMA expression in higher pTNM stages and tumor grades suggest that PSMA may be a good marker of biological aggressiveness suitable for patients' selection for more aggressive treatment. (Folia Histochemica et Cytobiologica 2013, Vol. 51, No. 1, $66-72)$
\end{abstract}

Key words: hTERT, telomerase, PSMA, serum PSA, immunohistochemistry, prostatic cancer

Corespondence address: A. Gasinska

Department of Applied Radiobiology, Centre of Oncology, Garncarska St. 11, 31-115 Krakow, Poland;

tel.:48 124231073 ;

fax: 481242266 80;

e-mail: z5gasins@cyf-kr.edu.pl

\section{Introduction}

Late prostate cancer $(\mathrm{PC})$ recognition is the main cause of treatment failure, similarly as in other neoplastic diseases. Earlier recognition based on biological markers could be helpful in identification of biological differences in benign and malignant lesions, what could further help in precise indication for more aggressive post-operative treatment (chemo/radio- 
therapy). Prostate specific antigen (PSA) is now commonly regarded as an indicator of prostate cancer stage and cannot be regarded as an independent diagnostic or prognostic factor in prostate cancer as determinations of PSA serum levels have limitations - lack of specificity, elevation in benign disease and failure to detect a number of PSA-negative tumors [1]. Despite the considerable attention given to PSA as a screening test, the standard method for diagnosing PC is a transrectal ultrasound (TRUS) guided prostate biopsy. Unfortunately, conventional biopsy protocols have been found to have unacceptable high false-negative rates [2]. The contrast enhanced color Doppler ultrasound targeted biopsy and the magnetic resonance imaging have been devised to increase the specificity of the PC detection, however, these methods still present considerable rates of false-positive and false-negative results [3]. Thus, PC diagnosis in men with persistently increased PSA after a negative initial TRUS guided biopsy has become a great challenge for urologists and pathologists. Therefore, better prognostic markers are needed, which would allow for the differentiation between benign and malignant cases [4].

It was suggested that detection of telomerase presence might be one of such markers. Telomerase is a ribonucleoprotein enzyme that adds telomeric repeats (TTAGGG) into chromosomal ends using a segment of its own RNA component (hTR) as a template. Its catalytic subunit, reverse transcriptase component (hTERT), controls its activity and its expression is restricted to the cells that demonstrate telomerase activity [5]. Because of its ability to immortalize cancer cells, it plays a significant role in tumor growth [6]. Previously it was shown that telomerase was activated in a large majority of human cancer tissues, however, not in most normal tissues or in tissues adjacent to malignant or benign tumors [6]. Therefore, it was suggested that telomerase activity can be a useful marker for cancer detection [7]. It was also found that telomerase activity was detected in approximately $90 \%$ of human cancer tissues $[6,8$, 9], and the level of telomerase activity correlated with prostate cancer pathologic grade, suggesting that telomerase activity may be a marker of tumor's malignant potential [10-12].

Apart from tumor biopsy, telomerase activity and/ or hTERT expression was also detected in clinical materials such as body fluids, secretions, washes, brushings, blood, and fine needle aspirates [7]. To detect cell telomerase activity, different methods have been applied: in situ telomeric repeat amplification protocol (TRAP assay), the RT-polymerase chain reaction (PCR) of mRNA and hTERT immunohis- tochemistry. However, recent evidence shows that telomerase might play novel, extratelomeric roles, unrelated to the telomere length regulation in mediating cell survival and anti-apoptotic functions against various cytotoxic stresses which can have potentially important biological consequences, particularly in cancer cells $[13,14]$. Furthermore, it was demonstrated, that hTERT regulates transcriptional activity of a key cell cycle regulator, cyclin D1 [15].

Prostate-specific membrane antigen (PSMA) is a membrane protein expressed in all forms of prostate tissue, including carcinoma [16]. In contrast to other highly restricted prostate-related antigens such as PSA and prostatic acid phosphatase, which are secretory proteins, PSMA is an integral cell membrane protein. The PSMA protein has three major domains: internal, transmembrane and extracellular parts [17] and acts as a glutamate-preferring carboxypeptidase [18]. Although PSMA is primarily expressed in normal human prostate epithelium, it was found to be upregulated and strongly expressed in prostate cancer cells, including those of the metastatic state [19]. Immunohistochemical staining revealed a correlation between PSMA expression and severity of cancer [19]. PSMA expression allows for the identification of benign and malignant prostatic epithelium and may be a potentially valuable marker in the treatment of patients with prostate cancer [20].

PSMA has an internalization signal that allows for the internalization of the protein on the cell surface, which makes it a useful antigenic target for therapy and diagnosis [21-23]. Till now, no correlation of telomerase or PSMA activity with preoperative serum PSA concentrations or pathologic data, including tumor grade and stage has been established. Therefore, we sought to evaluate their potential role as immunohistochemical diagnostic markers for patients with PC.

\section{Material and methods}

Patients. The study involved evaluation of 140 consecutive radical prostatectomy specimens, obtained from patients who underwent radical prostatectomy for localized PC between 2007 and 2011. The mean age of the patients was $62.9 \pm 6.2$ years. Clinical staging was carried out according to American Joint Committee on Cancer (AJCC) and the International Union Against Cancer [24]. Grading was established according to the Gleason score (GS) differentiation system (range 2-10) [25]. Patients were divided into three Gleason score groups according to American Joint Committee on Cancer (AJCC): well differentiated (Gleason score 6), moderately differentiated tumors (Gleason score of 7) and poorly differentiated tumors (Gleason score 8-10). The protocol was approved by the Ethical Commit- 

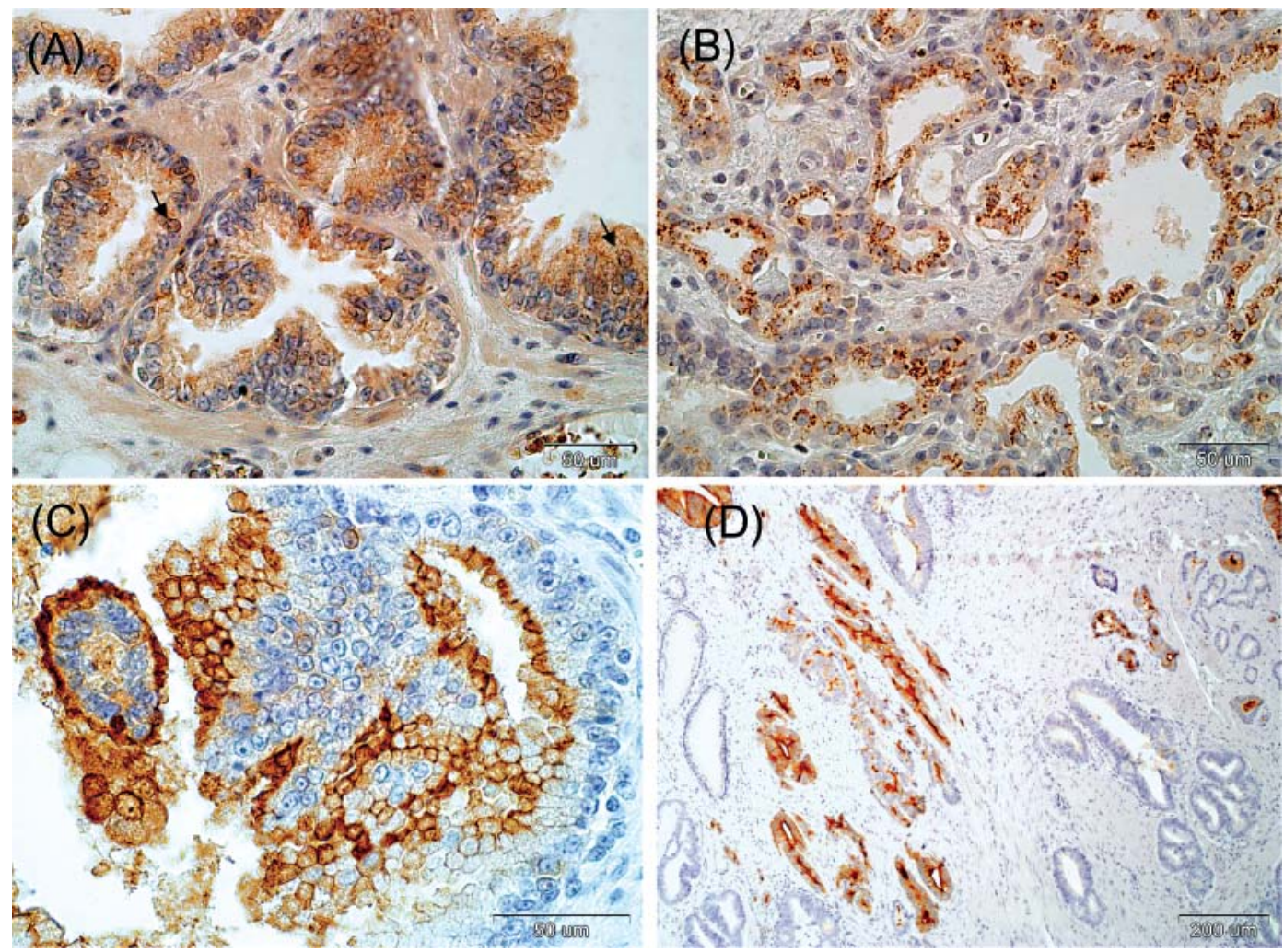

Figure 1. Immunohistochemical localizations of telomerase and PSMA in prostate cancer (PC). (A) Diffuse nuclear hTERT staining (positive, arrow) in low-differentiated and (B) cytoplasmic (not analyzed) staining in moderatelydifferentiated PC. (C) Strong PSMA immunoreactivity in infiltrating low-differentiated and (D) moderately differentiated PC. Note negative staining in the hyperplastic acini

tee of the Center of Oncology, and each patient submitted written consent.

Immunohistochemical assessment of tumor markers. Following rehydration, in $5 \mu \mathrm{m}$ sections heat-based antigen retrieval was carried out (for telomerase, $30 \mathrm{~min}$ at $96^{\circ} \mathrm{C}$ with Target Retrieval Solution, $\mathrm{pH}$ 9.0, for PSMA 20 min, $\mathrm{pH}$ 6.0). After cooling for 20 minutes, endogenous peroxidases were blocked using $0.3 \%$ hydrogen peroxide in $100 \%$ methanol. Next, the sections were washed, flooded with $2.5 \%$ normal horse blocking serum for $20 \mathrm{~min}$ and incubated with proper antibody overnight at $4^{\circ} \mathrm{C}$. For hTERT it was a rabbit polyclonal antibody to human telomerase (Novus Lab. Biologicals, Littleton, USA), (1:300), and for PSMA it was a mouse anti-human prostate specific membrane antigen (Novocastra, Newcastle, United Kingdom) (1:200). After washing, slides were incubated in room temperature for $1 \mathrm{~h}$ with ImmPRESS Reagent Kit visualization system containing proper antibody (VECTOR LAB, Inc, Burlingame, USA). The sections were stained with diaminobenzidine (DAB), counterstained with hematoxylin, dehydrated and mounted. Negative control slides omitting the primary antibody were included in each run of stains. The positive con- trol for anti-TERT was squamous cell esophageal carcinoma, while for PSMA - benign hypertrophic prostatic glands served as an internal control. The intensity of immunostaining was evaluated by light microscopy at $400 \times($ hTERT $)$ and $200 \times($ PSMA) magnification. For telomerase, only diffuse nuclear or diffuse nuclear with nucleolar accentuation was considered as positive staining (Figure 1A); cytoplasmic hTERT immunoreactivity (Figure 1B) was regarded as negative. For PSMA, immunoreactivity was identified as membrane staining (extracellular portion of the antigen) (Figure 1C) with two different patterns of positive staining including apical and/or apical and cytoplasmic. The binding occurred in the epithelial cells of the prostate but not in the basal or stromal cells (Figure 1D). Nonhyperplastic and benign hyperplastic acini were not evaluated. Protein expression was shown in the form of labeling indices (LI) interpreted as a percentage of positively immunostained cells (brown). About 500-1000 cells were counted in several (4-6) malignant areas of tissue sections.

Statistical analysis. Statistical analysis was performed with STATISTICA ver. 9. Intergroup differences in the original data were tested with ANOVA test or Student's t test. P 

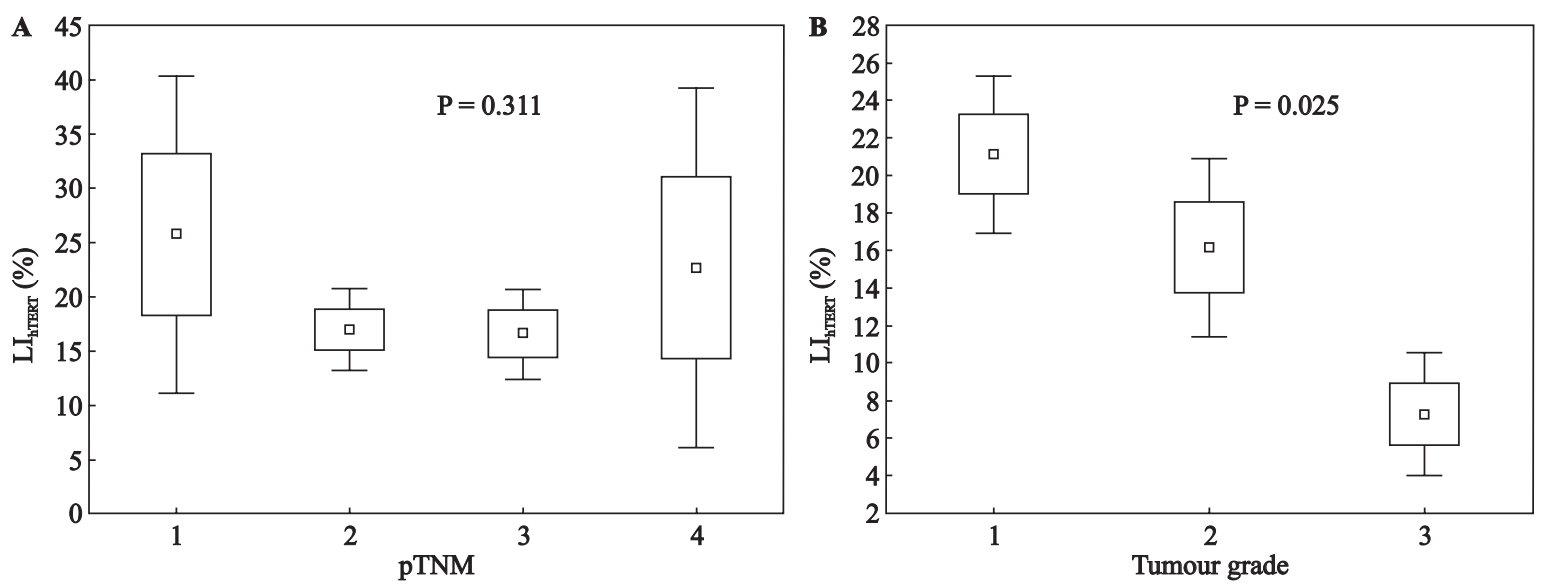

Figure 2. Association between telomerase immunoreactivity $\left(\mathrm{LI}_{\mathrm{hTERT}}\right)$ and $(\mathbf{A})$ pathological tumor stage $(\mathrm{pTNM})$ or $(\mathbf{B})$ tumor grade in prostate cancer. Symbols represent mean values $\pm \mathrm{SE}$
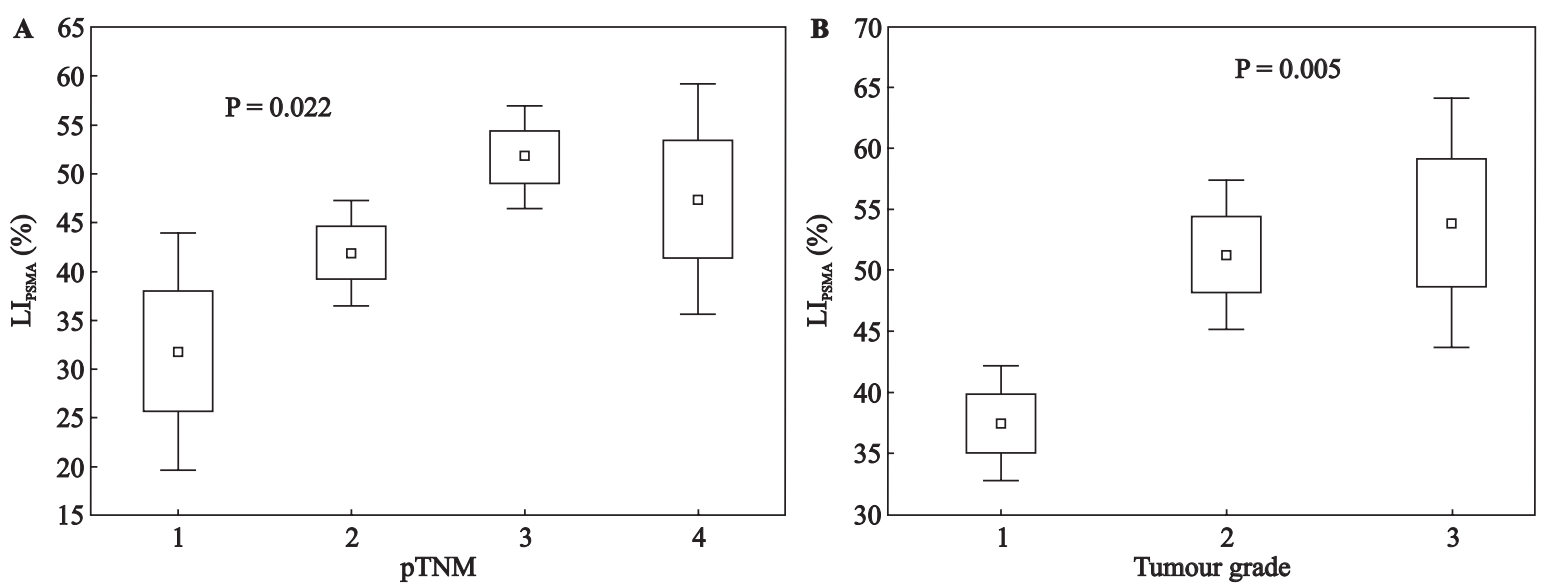

Figure 3. Association between PSMA expression $\left(\mathrm{LI}_{\mathrm{PSMA}}\right)$ and (A) pathological tumor stage (pTNM) or (B) tumor grade in prostate cancer. Symbols represent mean values $\pm \mathrm{SE}$

values of less than 0.05 were considered to indicate statistical significance.

\section{Results}

We examined human histological specimens from $140 \mathrm{PC}$ patients treated with radical surgery. There were 13 patients $(9.3 \%)$ at pTNM stage $1,78(55.7 \%)$ at stage 2, $40(28.6 \%)$ at stage 3 and $9(6.4 \%)$ at stage 4 , respectively. In the analyzed group there were $75(53.6 \%)$ well differentiated, $52(37.1 \%)$ moderately differentiated and $13(9.3 \%)$ poorly differentiated tumors. Pre-operative PSA revealed a median PSA of $9.9 \pm 5.5 \mathrm{ng} / \mathrm{mL}$. Concentration of serum PSA was significantly increased with pTNM stage $(\mathrm{P}=0.011)$, Gleason score $(\mathrm{P}=0.002)$, and tumor grade $(\mathrm{P}=0.003)$. In the three grade groups, nuclear reactivity of hTERT was present in $81 \%$ of sections and the mean
$\mathrm{LI}_{\mathrm{TERT}}$ was equal to $18.0 \pm 1.5 \%$. Expression of hTERT significantly decreased with tumor grade $(\mathrm{P}=0.025$, Figure 2). The mean LI ${ }_{\text {hTERT }}$ percentages for low to high-grade groups were $21.1 \%, 16.1 \%$ and $7.3 \%$, respectively. The number of positively staining hTERT cells was not correlated with Gleason score $(\mathrm{P}=0.142)$ and pathological tumor stage $(\mathrm{p}=0.311)$. In higher tumor grade, more frequently negative nuclear hTERT staining and positive hTERT localization in the cytosol was observed, and this form of staining was not taken into consideration.

For PSMA staining, in benign epithelium, weak cytoplasmic heterogeneous immunoreactivity was observed, however, only tumor cells were evaluated. The mean $\mathrm{LI}_{\mathrm{PSMA}}$ was $44.1 \pm 1.9 \%$. However, opposite results than in case of telomerase were observed. A positive correlation was demonstrated between the PSMA expression and Gleason score $(\mathrm{P}=0.003)$. 
Also PSMA expression statistically significantly correlated with pTNM stage $(\mathrm{P}=0.022$, Figure $3 \mathrm{~A})$ and grade $(\mathrm{P}=0.005$, Figure $3 \mathrm{~B})$. The mean $\mathrm{LI}_{\mathrm{PSMA}}$ value for low-grade PC was $37.5 \%$, for moderately differentiated tumors $51.3 \%$ and $53.9 \%$ for poorly differentiated tumors.

\section{Discussion}

We studied expression of two proteins thought to be prognostic biomarkers of malignancy in PC. This opinion can be true in case of PSMA for which we observed significant increase of expression with tumor stage and grade, whereas for telomerase, such a correlation was not found and therefore it cannot be considered as a good malignancy marker in PC. In our series, significant decrease of telomerase immunoreactivity (nuclear localization) with the tumor grade and tendency to lower values with tumor stage was observed. This was caused in high-grade tumors by translocation of positive hTERT staining from the nucleus, where its activity is connected with telomere maintenance, to the cytosol. As cytoplasmic hTERT activity is extratelomeric, this localization was not taken into consideration in our study. We used the same criteria as other authors analyzing earlier hTERT activity in PC where nucleolar /nuclear localization was considered as positive, whereas all cytoplasmic reactivity was regarded as negative [11]. It was shown earlier that telomerase activation occurs in the progress of carcinogenesis, hTERT mRNA was cytoplasmic and TERT protein was mainly found in the nucleus, indicating that TERT protein produced by the transcription of mRNA in the cytoplasm becomes localized in the nucleus [11]. However, our results contradict the studies based also on immunohistochemistry showing the greater percentage of hTERT positive cells in high-grade than low-grade prostatic cancers $[11,26]$ and displaying stronger hTERT activity in higher cancer stages [12]. In our study, the relation was reverse. We observed non-significant decrease in the immunoreactivity of the enzyme with pathological stage and Gleason score, what may be in agreement with the findings of others [9, 27]. Additionally, in our study, the mean LI ${ }_{\text {hTERT }}$ for different grade groups was two-times [11] and threetimes [26] lower than in the studies of other authors what indicates that the results are controversial. The reason for this discrepancy might be not only methodological differences: different monoclonal antibodies used and the different number of analyzed tumors (much smaller number of high-grade tumors in our study), but also the fact that some telomerase functions were not fully recognized at that time.
Recently, a new aspect of telomerase activity, independent of telomeres lengthening has emerged to explain its protective effects on cell survival and stress resistance [13]. The observation that TERT resides not only in the nucleus, but also in the cytosol, reinforces the notion of possible telomere-independent functions [13]. It has been suggested that telomerase in the cytoplasm protects mitochondria from oxidative stress [28] and performs other extratelomeric functions like enhancing cell viability under conditions of environmental stress such as hypoxia [29]. Telomerase has been shown to shuttle dynamically between different cellular locations. Under increased oxidative stress, telomerase is excluded from the nucleus and can be found within the mitochondria [30] that seem to be unrelated to telomere maintenance. This phenotype (with cytoplasmic TERT localization) correlates with decreased oxidative stress and improved mitochondrial function by currently largely unknown mechanisms [28-30]. These data correlate with pro-survival and anti-apoptotic functions of telomerase. This may be in agreement with our results obtained in the analysis of the same group of tumors showing high proliferative potential (based on Ki-67) and active glycolytic metabolism (GLUT-1 expression) in high-grade PC tumors [31]. Although our understanding of the underlying mechanisms is limited and the biological significance of these processes requires further investigation, the data add to the already established non-canonical functions of telomerase that correlate with its extra-nuclear localization. Nowadays, it is considered that transport of hTERT to the mitochondria seems to be an induced, directed and naturally occurring process [30]. According to Maser et al. [32] telomerase is located within the nucleolus or the cytoplasm and only gets targeted to the telomere during replication. All these observations suggest that those novel telomerase functions work by mechanisms that are separated from normal telomere lengthening and capping, considered in the past to be the main feature of malignancy. Therefore, these, not yet sufficiently known, extra-telomeric telomerase functions, independent of catalytic activity, require establishing new assessment criteria of the enzyme «s immunoreactivity before it is recommended as a marker of malignancy in clinical setting.

Androgens play a central role in prostate development and prostate cancer proliferation [33]. Several reports suggest that telomerase activity is regulated by androgens in vivo and low androgen level might create problems with translocation of hTERT protein from cytoplasm to the nucleus [33, 34]. This may be in agreement with the study showing that androgen ablation reduces telomerase most dramatically in the highest grade 
malignancies [35] and decreases PSA serum levels because testosterone which is required to drive production of PSA is surgically or medically eliminated [36].

In our study, concentration of serum PSA positively correlated with pTNM stage, Gleason score, and tumor grade. PSA is a glycoprotein secreted into the lumen of prostatic glands to liquefy the seminal coagulation. In invasive adenocarcinomas, disruption of the normal glandular architecture and loss of the polarity of prostatic cells appear to allow increased direct PSA diffusion to prostate gland vessels.

In the present study, the increased expression of PSMA correlated significantly with higher preoperative PSA levels, high tumor grade and advanced tumor stage. Although PSA and PSMA are regulated differentially, their expression showed a correlation with malignant transformation. In our series, the expression of PSMA was significantly greater in higher tumor advancement and grade and was larger than that of PSA in poorly differentiated tumors, which confirms earlier studies [19]. Our finding is consistent with previous reports using immunohistochemistry and multiplex PCR reaction to demonstrate the association between PSMA and tumor progression $[37,38]$. The mean $\mathrm{LI}_{\mathrm{PSMA}}$ value in our study was $44.1 \%$ and was within the range $(30-100 \%)$ given by different authors $[19,20,36,37]$. The difference in the mean values might be caused by non-equal number of cases in the analyzed tumor grade groups (value ranges were similar).

In normal and hyperplastic prostate epithelium we observed weak and heterogeneous positive PSMA expression which is in agreement with other authors' observations [19, 20, 27]. In normal prostate tissue, the dominating form of this protein is the one that appears in the cytoplasm. However, in PC cells PSMA expression was mainly membranous. Low expression in normal hyperplastic tissue would suggest a limited role of PSMA in normal human prostate or hyperplastic tissue. It was shown that PSMA expression was negatively regulated by androgen levels [36]. The increased expression of PSMA in poorly differentiated tumors suggests that this protein may be associated with development of hormone-independent prostate cancers [36]. Furthermore, the enhanced expression of PSMA occurs after androgen deprivation what suggests that PSMA is upregulated in the majority of PC after androgen treatment. Therefore, assessment of PSMA expression may be helpful in indication for more aggressive treatment (chemo-/radiotherapy).

The prostate-restricted nature of PSMA, coupled with the direct association between the level of PSA expression and increasingly aggressive disease, implies a potentially important role of PSMA in PC biology.
This may confirm the study of Rajasekaran and coworkers [39] who indicated that PSMA has a causal role in the induction of aneuploidy and might play an etiologic role in the PC progression.

PSMA, as an integral membrane protein highly specific for prostate, may prove to be effective as a target for imaging and cytotoxic targeting modalities $[23,40]$. Monoclonal antibodies may be internalized by PC cells [21]. The availability of prostate-specific internalizing antibodies should aid the development of novel therapeutic methods to target the delivery of toxins, drugs, or short-range isotopes specifically to the interior of prostate cancer cells $[22,23,40]$. The high expression in metastatic tissues strongly suggests that PSMA may be a clinically useful target for antibody and genetic-directed therapy of PC that recurs after androgen deprivation [36].

\section{Conclusions}

Our data confirm that PSMA expression allows the identification of benign and malignant prostatic epithelium and may be a potentially valuable marker in the treatment of prostatic cancer. The export of hTERT from nucleus to the cytoplasm observed in high grade PC may suggest that telomerase plays a different role than telomere lengthening in prostate cancer. Before the assessment criteria are established, evaluation of hTERT immunoreactivity cannot be recommended as a biomarker of malignancy.

\section{Acknowledgments}

This work was supported by the Polish Ministry of Science and Higher Education (grant No N403 240837).

\section{References}

1. Bickers B, Aukim-Hastie C. New molecular biomarkers for the prognosis and management of prostate cancer - the post PSA era. Anticancer Res. 2009;29:3289-3298.

2. Welch HG, Fisher ES, Gottlieb DJ, Barry MJ. Detection of prostate cancer via biopsy in the medicare-SEER population during the PSA era. J Natl Cancer Inst. 2007;99:1395-1400.

3. Yuen JS, Thng CH. Endorectal magnetic resonance imaging and spectroscopy for detection of foci in men with prior negative transrectal ultrasound prostate biopsy. J Urol. 2004;171: 1482-1486.

4. Bjartell A, Montironi R, Berney MD, Egevad L. Tumor markers in prostate cancer II: Diagnostic and prognostic cellular biomarkers. Acta Oncol. 2011;50: 76-84.

5. Counter Ch M, Meyerson M, Eaton EN, Ellisen LW, Dickinson Caddl, Haber DA et al. Telomerase activity is restored in human cells by ectopic expression of hTERT (hEST2), the catalytic subunit of telomerase. Oncogene 1998;16:1217-1222.

6. Kim NW, Piatyszek MA, Prowse KR, Harley CB, West MD, Ho PLC et al. Specific association of human telomerase activity with immortal cells and cancer. Science 1994;266:2011-2015. 
7. Hiyama E, Hiyama K. Telomerase as tumor marker. Cancer Lett. 2003;194:221-233.

8. Lin Y, Uemura H, Fujinami K, Hosaka M, Harada M, Kubota Y et al. Telomerase activity in primary prostate cancer. J Urol. 1997;157:1161-1165.

9. Zhang W, Kapusta LR, Slingerland JM, Klotz LH. Telomerase activity in prostate cancer, prostatic intraepithelial neoplasia, and benign prostatic epithelium. Cancer Res. 1998;58:619-621.

10. Sommerfeld HJ, Meeker AK, Piatyszek MA, Bova GS, Shay JW, Coffey DS. Telomerase activity: a prevalent marker of malignant human prostate tissue. Cancer Res. 1996;56:218-222 .

11. Iczkowski KA, Pantazis CG, McGregor DH, Wu Y, Tawfik OW. Telomerase reverse transcriptase subunit immunoreactiviy. A marker for high-grade carcinoma. Cancer 2002;96: 2487-2493.

12. Wang Z, Ramin SA, Tsai C, Lui P, Ruckle HC, Beltz RE et al. Telomerase activity in prostate sextant needle cores from radical prostatectomy specimens. Urol Oncol. 2001;6:57-62.

13. Chung HK, Cheong Ch, Song J, Lee HW. Extratelomeric functions of telomerase. Curr Mol Med. 2005;5:233-241.

14. Sung YH, Choi YS, Cheong C, Lee HW. The pleiotropy of telomerase against cell death. Mol Cells 2005;19:303-309.

15. Jagadeesh S, Banerjee PP. Telomerase reverse transcriptase regulates the expression of a key cell cycle regulator, cyclin D1. Biochem Biophys Res Commun. 2006;347:774-780.

16. Israeli RS, Powell CT, Fair WR, Heston WDW. Molecular cloning of a complementary DNA encoding a prostate-specific membrane antige. Cancer Res. 1993;53:227-230.

17. Chang SS. Overview of prostate-specific membrane antigen. Rev Urol. 2004;6 (suppl 10) S13-S18.

18. Pinto JT, Suffoletto BP, Berzin TM, Qiao H, Lin S, Tong WP et al. Prostate-specific membrane antigen: a novel folate hydrolase in human prostatic carcinoma cells. Clin Cancer Res. 1996;2:1445-1451.

19. Bostwick DG, Pacelli A, Blute M, Roche P, Murphy GP. Prostate specific membrane antigen expression in prostatic intraepithelial neoplasia and adenocarcinoma. A study of 184 cases. Cancer 1998;82:2256-2261.

20. Sweat SD, Pacelli A, Murphy GP, Bostwick DG. Prostatespecific membrane antigen expression is greatest in prostate adenocarcinoma and lymph node metastases. Urology 1998:52:637-640.

21. Liu H, Rajasekaran AK, Moy P, Xia Y, Kim S et al. Constitutive and antibody-induced internalization of prostate-specific membrane antigen. Cancer Res.1998;58:4055-4060.

22. Liu T, Wu LY, Berkman CE. Prostate-specific membrane antigen-targeted photodynamic therapy induces rapid cytoskeletal disruption. Cancer Lett. 2010;296:106-112.

23. Osborne JR, Akhtar NH, Vallabhajosula S, Anand A, Deh $\mathrm{K}$ et al. Prostate-speciifc membrane antigen-based imaging. Urol Oncol. 2012;31:144-154.

24. Edge SB, Byrd DR, Compton CC, Fritz AG, Greene FL, Trotti A. AJCC Cancer Staging Manual, 7th ed.; New York: Springer; 2010:457-464.
25. Gleason DF. The Veterans Administration Cooperative Urological Research Group: histological grading and clinical staging of prostatic carcinoma. In: Tannenbaum M, ed. Urologic Pathology: The prostate. Philadelphia: Lea and Febiger; 1997:171-197.

26. Puebla-Mora AG, Heras A, Cano-Valdez AM, Dominiquez-Malagon H. Human telomerase and ?-methyl-coenzyme A racemase in prostatic carcinoma. A comparative immunohistochemical study. An Diagn Pathol. 2006;10:205-208.

27. Latil A, Vidaud D, Valeri A, Fournier G, Vidaud M et al. hTERT expression correlates with $M Y C$ over-expression in human prostate cancer. Int J Cancer. 2000:89:172-176.

28. Ahmed S, Passos JF, Birker MJ, Beckmann T, Brings S. Telomerase does not counteract shortening but protects mitochondrial function under oxidative stress.J Cell Sci. 2008;121: 1046-1053.

29. Bollmann FM. The many faces of telomerase: emerging extratelomeric effects. BioEssays. 2008;30:728-732.

30. Saretzki G. Telomerase, mitochondria and oxidative stress. Exp Geront. 2009;44:485-492.

31. Luczynska E, Gasinska A, Wilk W. Expression of Ki-67 (MIB-1) and GLUT-1 proteins in non-advanced prostatic cancer. Pol J Pathol. 2012;63 272-277.

32. Maser RS, DePinho RA. Keeping telomerase in its place. Nature Med. 2002;8:934-936.

33. Wang Y, Kreisberg JI, Ghosh PM. Cross-talk between the androgen receptor and the phosphatidylinositol 3-kinase/Akt pathway in prostate cancer. Curr Cancer Drug Targets. 2007;7: 591-604.

34. Moehren U, Papaioannou M, Reeb Ch, Grasselli A, Nanni $\mathrm{S}$, Asim $\mathrm{M}$ et al. Wild-type but not mutant androgen receptor inhibits expression of the hTERT telomerase subunit: a novel role of AR mutation for prostate cancer development. FASEB J. 2008;22:1258-1267.

35. Iczkowski K, Huang W, Mazzucchelli R, Pantazis CG, Stevens GR, Montironi R. Androgen ablation therapy for prostate carcionoma suppresses the immunoreactive telomerase subunit hTERT. Cancer. 2004;100:294-299.

36. Wright GL, Grob MB, Heley C, Grossman, Newhall K, Petrylak D et al. Upregulation of prostate-specific membrane antigen after androgen-deprivation therapy. Urology 1996;48:326-334.

37. Jemaa A, Bouraoui Y, Sallami S, Banasr A, Ben Rais N, Quertani L et al. Co-expression and impact of prostate specific membrane antigen and prostate specific antigen in prostatic pathologies. J Exp Clin Cancer Res. 2010; 29: 171-180.

38. Perner S, Hofer MD, Kim R, Moller P, Hautmann RE et al. Prostate-specific membrane antigen expression as a predictor of prostate cancer progression. Hum Pathol. 2007;38:696-701.

39. Rajasekaran SA, Christiansen JJ, Schmid I, Oshima E, Sakamoto K, Weinstein J et al. Prostate-specific membrane antigen associates with anaphase-promoting complex and induces chromosomal instability. Mol Cancer Ther. 2008;7:2142-2151.

40. Akhtar NH, Pail O, Saran A, Tyrell L, Tagawa ST. Prostatespecific membrane antigen -based therapeutics. Adv Urol. 2012; doi:10.1155/2012/973820.

Submitted: 19 September, 2012 Accepted after reviews: 25 March, 2013 